\title{
Hyperthermic Intraperitoneal Chemotherapy After Cytoreductive Surgery; Experience and Short Term Outcomes
}



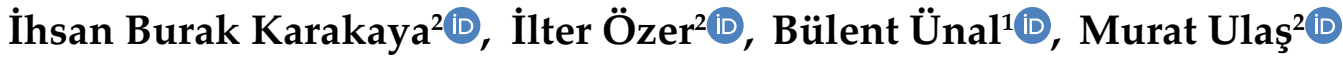

1 Eskişehir Osmangazi University Faculty of Medicine, Department of General Surgery, Section of Surgical Oncology, Eskişehir, Turkey

2 Eskişehir Osmangazi University Faculty of Medicine, Department of General Surgery, Section of Gastroenterological Surgery, Eskişehir, Turkey

\begin{abstract}
Background: Cytoreductive surgery + hyperthermic intraperitoneal chemotherapy is an important treatment option in patients with a primary diagnosis of colorectal cancer, ovarian cancer, appendix cancer, gastric cancer(selected cases), malignant peritoneal mesothelioma, and peritoneal pseudomyxoma in the presence of peritoneal involvement and resectable lesions limited to the abdomen. In this study, it was aimed to discuss cytoreductive surgery(CRS) with hyperthermic intraperitoneal chemotherapy(HIPEC) in the light of literature.

Methods: The data of patients who underwent cytoreductive surgery with hyperthermic intraperitoneal chemotherapy between June 2017 and September 2020 at our institution were analyzed. The study was designed retrospectively, and all patients who were discussed at the oncology council and decided on CRS + HIPEC were included in the study.

Results: 31 patients were included in the study. Primary diagnoses of the patients were colorectal cancer in 15 (48\%), ovarian cancer in $9(29 \%)$, stomach cancer in $3(10 \%)$, mesothelioma in $2(7 \%)$, appendix cancer in $1(3 \%)$, and also peritoneal pseudomyxoma in 1 (3\%). Therapeutic HIPEC was performed in 30 patients, and prophylactic HIPEC was performed in 1 patient. Cytoreduction score was 0 in all patients. The median peritoneal cancer index was 15 (7-29). the median number of resected organs was 3(1-6). Stoma formation was performed in 14 patients (45\%). During the postoperative 30 days, mortality was observed in 1 patient (3\%) and morbidity in 5 patients $(16 \%)$.
\end{abstract}

Conclusion: The early postoperative mortality and morbidity results reported in our study are compatible with those in the literature.

Keywords: Peritoneal Carcinomatosis, Cytoreductive Surgery, Hyperthermic Intraperitoneal Chemotherapy. 


\section{INTRODUCTION}

Peritoneal carcinomatosis (PC) is a clinical entity detected in advanced stages of gastrointestinal and gynecological cancers and primary ones of the peritoneum, such as peritoneal mesothelioma peritoneal pseudomyxoma, as well as gastrointestinal and gynecological cancer, and adversely affects long-term survival (1). With comprehending the intraperitoneal spread biology of tumors in the last thirty years, PC has been accepted as a local-regional disease. $10-35 \%$ of colorectal cancer recurrences and up to $50 \%$ of gastric cancer recurrences are limited only in the peritoneal cavity. On the other hand, $15 \%$ of colorectal cancer patients and almost $40 \%$ of gastric cancer patients have peritoneal carcinomatosis during perioperative exploration (2). Malignant peritoneal mesothelioma (MPM), which constitutes 30\% of all mesothelioma tumors, is a fatal disease with an average overall survival of $6-12$ months (3). Epithelial ovarian cancer (EOC), a fatal type of gynecological cancer, is responsible for approximately $50 \%$ of the mortality from gynecological cancers. Serous adenocarcinoma is the most common subtype of epithelial ovarian cancer. Approximately $95 \%$ of the patients are diagnosed with the disease spreading beyond the ovaries (Stage II-IV) and has a poor prognosis. Many patients with diffuse peritoneal disease in EOC develop intraperitoneal recurrence despite of complete cytoreduction and chemotherapy. Alternative methods for increasing the effectiveness of chemotherapy have been investigated (4).

Cytoreductive surgery (CRS), which was firstly described by Sugarbaker, includes organ resection and peritonectomy procedures that leave no visible lesions within the intraabdominal cavity (5). Hyperthermic intraperitoneal chemotherapy (HIPEC) is performed for the curative treatment of invisible microscopic residual tumor deposits that may remain after CRS $(4,6)$. CRS + HIPEC is indicated for peritoneal metastases of peritoneal pseudomyxoma, malignant peritoneal mesothelioma, colorectal and ovarian cancers. CRS + HIPEC is in the developmental stage in peritoneal metastases of gastric cancer, and it is not used for pathologies such as sarcoma and GIST (7). Although CRS + HIPEC is a technique that has high morbidity and mortality, appropriate patient selection and development of multi-modal treatment options have improved the outcomes to better levels in the last two decades. As a result of these improved outcomes, it was observed that multidisciplinary oncology councils had increased referral of patients with peritoneal carcinomatosis to surgery who are eligible for CRS + HIPEC (8).

In this study, we aimed to present the demographic, clinicopathological characteristics and early postoperative morbidity, mortality results of the patients who underwent CRS + HIPEC in the light of the literature.

\section{MATERIALS AND METHODS}

The data of patients who underwent cytoreductive surgery with hyperthermic intraperitoneal chemotherapy between June 2017 and September 2020 at our institution were analyzed. The study was designed retrospectively, and all patients who were discussed at the oncology council and decided on CRS + HIPEC were included in the study. The oncology council decided the operation for patients with no signs of extra-abdominal solid organ metastasis in thoracoabdominal CT imaging and sufficient cardiorespiratory and renal function to tolerate aggressive procedures. Patients with Eastern Cooperative Oncology Group (ECOG) performance scores above two were excluded from the study (9). The study was conducted in accordance with the principles of the Declaration of Helsinki. Data of patients regarding age, gender, pathological findings, cytoreduction score (CC), peritoneal cancer index (PCI), perioperative complications, length of hospital stay, recurrence, and follow-up were recorded.

The distribution and extent of tumor deposits in 13 abdominopelvic regions were evaluated and recorded as PCI during abdominal exploration. PCI is scored according to the size of the tumor nodules as follows: lesion size(LS)-0: no tumor; LS-1: tumor nodule $<0.5 \mathrm{~cm}$; LS-2: 0.5-5 cm tumor nodule; and LS-3: tumor nodule $>5$ $\mathrm{cm}$. The patients were divided into two groups according to their PCI scores as less than 20 and more.

Completeness of cytoreduction (CC) was classified by the surgeon for each patient using Sugarbaker's criteria (10). After cytoreduction, completeness of cytoreduction (CC) was determined as follows; CC-0, no visible evidence of disease; CC-1, tumor deposits are less than $2.5 \mathrm{~mm}$ in diameter, without a confluence of disease at any site; CC2, tumor deposits are between $2.5 \mathrm{~mm}$ and $2.5 \mathrm{~cm}$ and the absence of a contiguous layer of disease at any anatomic site in the abdomen or pelvis; CC-3, tumor deposits are greater than $2.5 \mathrm{~cm}$ in diameter or a confluence of disease layered out at any site within the abdomen or pelvis. The 
parietal peritoneum, tumor-involved visceral peritoneum, and tumor-involved gastrointestinal tract should be resected in the CRS procedure. If tumor implants are observed, cholecystectomy, segmental liver resection, splenectomy, hysterectomy, and bilateral salpingooophorectomy should be performed if possible.

In our study, HIPEC was performed just after SRC using a hyperthermia pump with a closed technique. The peritoneal perfusate was warmed to $42^{\circ} \mathrm{C}$ and infused into the abdomen with $1000 \mathrm{cc} / \mathrm{min}$. The perfusate at 42$43{ }^{\circ} \mathrm{C}$ containing chemotherapeutic agents was rotated in the intraperitoneal area for 60 minutes. After the HIPEC procedure, all catheters and temperature probes were removed. After the perfusion and rotation, the abdominal cavity was opened again and washed with 5000 cC saline solution. Gastrointestinal anastomoses (whether manually or with a stapler) were performed after the HIPEC procedure. All procedures were performed by the same surgical team that has high experience in regional treatments.

Complications were recorded according to the ClavienDindo system (grade 1:mild complications, grade 5:death) (11). Operative mortality was defined and recorded as any death within 30 days of surgery.

During the first two years, a postoperative follow-up which includes a physical examination, thoracoabdominal CT imaging, and plasma tumor marker levels, was evaluated every three months. These evaluations were then repeated every six months for three years. Recurrent disease or progression was confirmed pathologically.

Statistical analysis was performed using the statistical software package SPSS version 25.0 (IBM Corp., Armonk, NY, USA). Binary variables were reported as counts and percentages. The Kaplan-Meier test was used to identify differences between curves. Survival was measured from the time of diagnosis.

This study was approved by the Eskişehir Osmangazi University non-invasive clinical research ethics committee with the date of 12.01.2021 and number of 7. The aforementioned study was conducted according to a recent version of the declaration of Helsinki.

\section{RESULTS}

There were 31 patients in the study. The median age of the patients was 55(37-76) years. 20 patients were women and 11 patients were men. Demographic and clinicopathological characteristics are shown in Table 1. Primary diagnoses of the patients were $15(48 \%)$ colorectal cancer, 9 (29\%) ovarian cancer, $3(10 \%)$ stomach cancer, $2(7 \%)$ mesothelioma, $1(3 \%)$ appendix cancer and 1 (3\%) peritoneal pseudomyxoma (figure-1). 14 patients had abdominal distension, 4 patients had abdominal pain, 2 patients had ileus. Peritoneal carcinomatosis or ascites were demonstrated radiologically in all patients. Pathological verification was not carried out for the diagnosis of peritoneal carcinomatosis or ascites, except for mesothelioma and peritoneal pseudomyxoma. All procedures were performed via laparotomy. 30 patients underwent therapeutic HIPEC and 1 patient underwent prophylactic HIPEC. The patient who underwent prophylactic HIPEC had limited peritoneal involvement and ascites in diagnostic laparotomy. An implant in the peritoneum was excised and sent to the pathology laboratory for frozen examination. Frozen examination was reported as adenocarcinoma metastasis. Therefore, the operation was terminated and the patient was referred to the medical oncology clinic for systemic chemotherapy. The patient's condition after systemic chemotherapy was discussed in the oncology council. Oncology council decided to perform prophylactic HIPEC operation together with gastrectomy. CC score was 0 in all patients. The median PCI was 15 (7-29)(Figure-2). The median number of resected organs was 3 (1-6). Stoma formation was performed in 14 patients (45\%). The rate of intraperitoneally applied chemotherapeutics were as follows; Oxaliplatin in 16 patients $(51 \%)$, cisplatin in 7 patients $(22 \%)$, mitomycin in 3 patients $(10 \%)$, mitomycincisplatin in 2 patients $(7 \%)$, doxataxel-oxaliplatin in 2 patients $(7 \%)$ and 1 patient $(3 \%)$ cisplatin- doxataxel (Figure-3). 
Table 1. Demographic and clinicopathological features

\begin{tabular}{|l|l|}
\hline Features & Values, (\%) \\
\hline Age (year) mean & $55(37-76)$ \\
50> & $8(25 \%)$ \\
$50<$ & $23(75 \%)$ \\
\hline Gender & \\
Male & $11(35 \%)$ \\
Female & $20(65 \%)$ \\
\hline Location & \\
Colorectal & $15(48 \%)$ \\
Over & $9(29 \%)$ \\
Stomach & $3(10 \%)$ \\
Mesothelioma & $2(7 \%)$ \\
Appendix & $1(3 \%)$ \\
Peritoneal pseudomyxoma & $1(3 \%)$ \\
\hline PCI & $15(7-29)$ \\
20> & $23(75 \%)$ \\
20< & $8(25 \%)$ \\
\hline CC & \\
Score 0-1 & $31(100 \%)$ \\
Score 2-3 & 0 \\
\hline Differentiation & \\
Well & $12(44 \%)$ \\
Moderate & $5(19 \%)$ \\
Poor & $10(37 \%)$ \\
\hline Obstruction & \\
Present & $2(7 \%)$ \\
Absent & $25(93 \%)$ \\
\hline Resection of organ (number) mean & $3(1-6)$ \\
\hline Stoma & \\
Yes & $14(45 \%)$ \\
No & $17(55 \%)$ \\
\hline Protocol of HIPEC & \\
Oxaliplatin & $16(51 \%)$ \\
Cisplatin & $7(22 \%)$ \\
Mitomycin & $3(10 \%)$ \\
Doxorubicin+Oxaliplatin & $2(7 \%)$ \\
Mitomycin+Cisplatin & $2(7 \%)$ \\
Cisplatin +Doxorubicin & $1(3 \%)$ \\
\hline Perioperative mortality & $1(3 \%)$ \\
\hline Perioperative morbidity & \\
Grade 1-2 & \\
Grade 3-4-5 & $4(13 \%)$ \\
\hline Complications & $1(3 \%)$ \\
Leakage of Anastomosis \\
perforation of intestine & $1(3 \%)$ \\
Evisceration & $1(3 \%)$ \\
Bleeding & $1(3 \%)$ \\
Fealure of kidney & $1(3 \%)$ \\
\hline Lenght of Stay (day) mean & $1(3 \%)$ \\
7z & \\
$7<$ & \\
\hline Abbreviation:CC=Score ofCytoreduction;PCI $=$ Peritoneal \\
carcinomatosis Index
\end{tabular}

Figure 1. Diseases

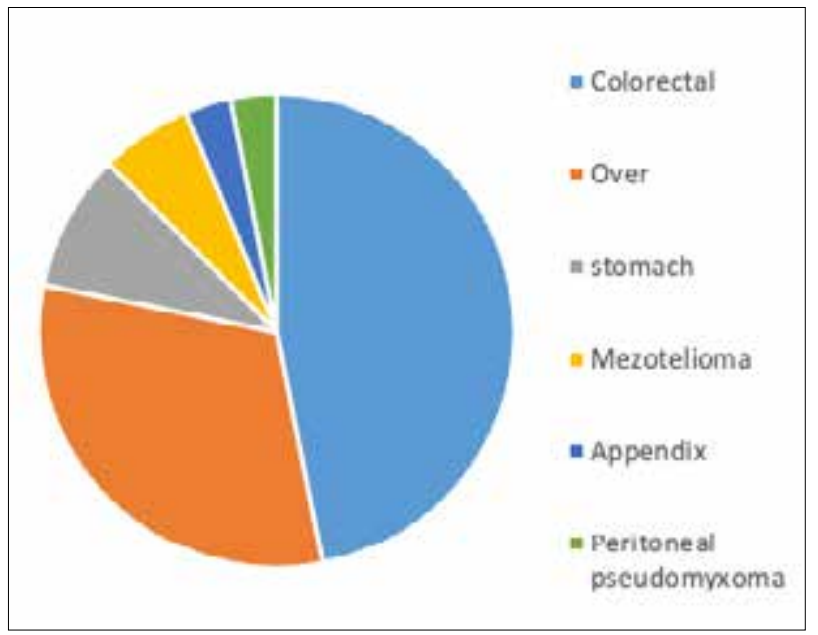

Figure 2. Peritoneal Carcinomatosis Index

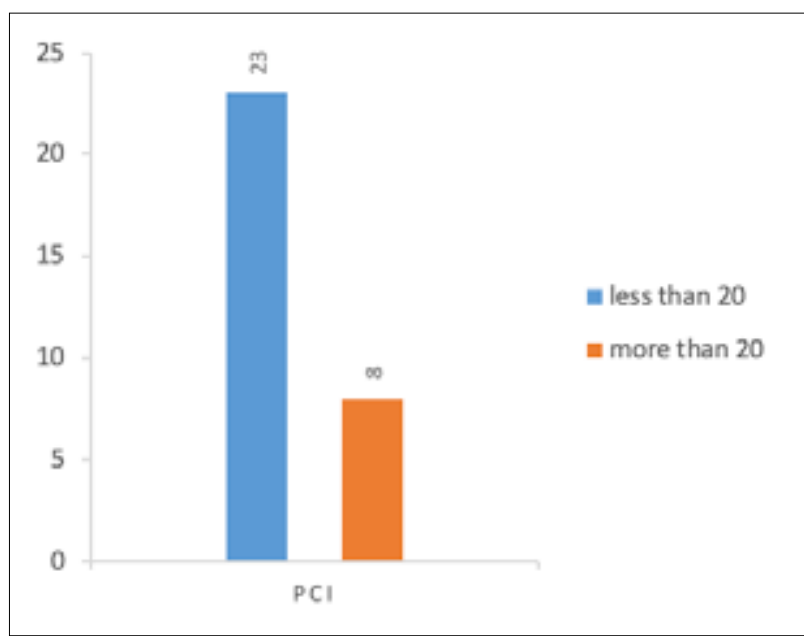

Figure 3. Protocol of HIPEC

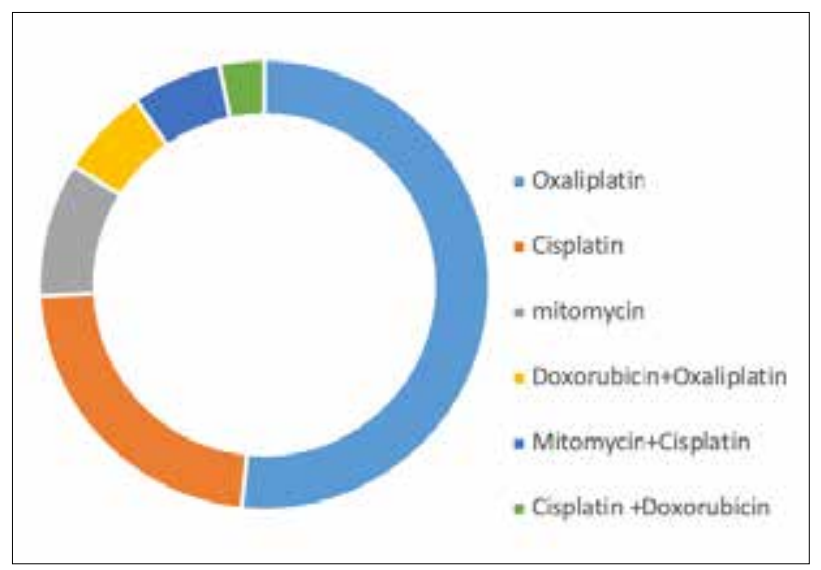

Median length of hospitalisation was 10 (5-24) days. ECOG performance status of all patients in the study was 0 or 1 . In the postoperative first 30 days, mortality and 
morbidity rates were $1(3 \%)$ and $5(16 \%)$, respectively. In this patient, prophylactic CRS + HIPEC was performed in order to gastric cancer. In the postoperative follow-up, after anastomotic leak, the patient died as a result of septic shock and multiorgan failure. Other complications are presented in Table 1.

The overall mortality rate in our series was $19 \%$. The mean follow-up period was 20 (1-36) months. The mean overall survival time was 22 months. At 12 and 24 months, the survival results were $82 \%$ and $78 \%$ for overall survival, and $69 \%$ and $48 \%$ for disease free survival, respectively (Figure-4, Figure-5).

Figure 4. Overall survival of 31 patients with SRCHIPEC according to the Kaplan-Meier method

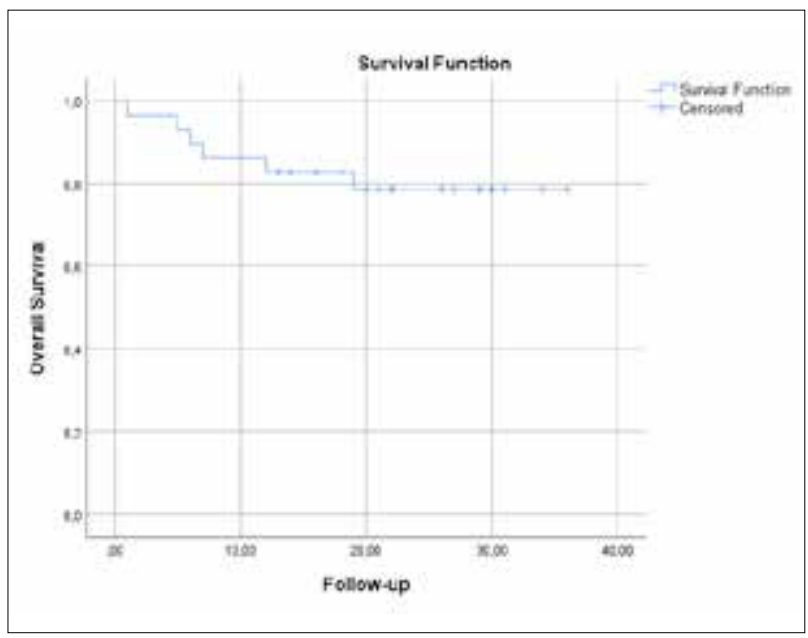

Figure 5. Disease free survival of $\mathbf{3 1}$ patients with SRCHIPEC according to the Kaplan-Meier method

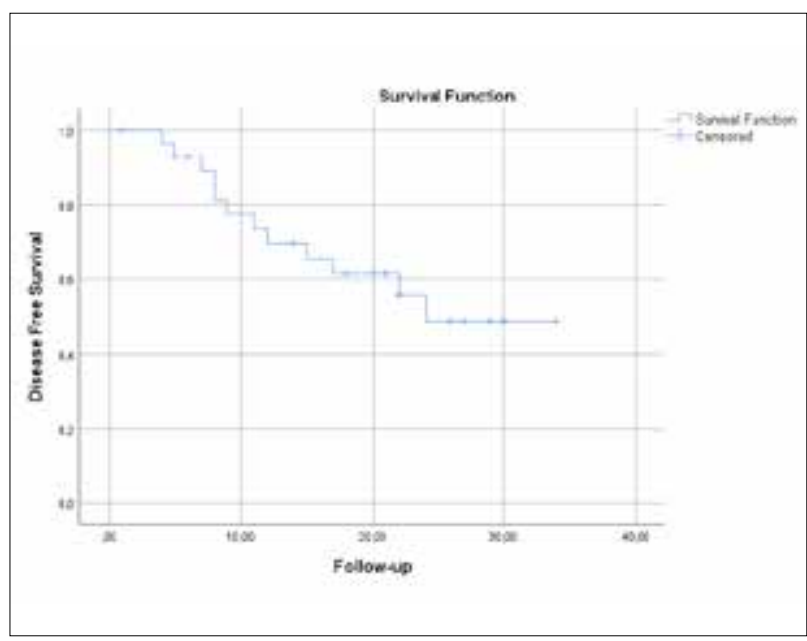

\section{DISCUSSION}

Peritoneal carcinomatosis(PC) is a entity with high mortality and low survival. The benefit of systemic chemotherapy is limited. CRS + HIPEC has better results compared to conventional systemic chemotherapy response in diseases such as colorectal cancer, gynecological cancers(especially ovarian cancer), peritoneal carcinomatosis due to appendix cancer, peritoneal mesothelioma and peritoneal pseudomyxoma. CRS + HIPEC procedure is in development stage in stomach cancer, studies regarding its effectiveness are ongoing (12). Delivering chemotherapeutic agents directly to the abdominal cavity via a catheter, provides a high concentration of drug at the peritoneal surface, thus enhances the cytotoxic effect of chemotherapy. However hyperthermia has a direct cytotoxic effect on tumor cells and also increases the effect of chemotherapeutic agent. Philosophy of HIPEC is based on these two basic ideas (4). Tissue penetration depth of chemotherapeutics is up to 1-2 $\mathrm{mm}$. Therefore, it is accepted that patients who do not have macroscopic tumors after cytoreductive surgery are more likely to benefit from HIPEC. This opinion is the main source of the concept for HIPEC after maximal CRS (1). Patient selection was carried out by carefully examining clinical and radiological findings in the multidisciplinary oncology council. For this reason, our rate of complete cytoreduction was high.

In the preoperative period, imaging methods can contribute to the diagnosis of $\mathrm{PC}$ and to determine its severity. The sensitivity of computed tomography (CT) is $33 \%$ and its specificity is over $90 \%$. The sensitivity of ultrasonography is $9 \%$. In PC due to stomach cancer, endosonography (EUS) is more sensitive than CT. Magnetic resonance (MRI) provides better soft tissue contrast agent resolution than $\mathrm{CT}$, furthermore there is no risk of contrast agent nephropathy and ionizing radiation. However, a significant superiority to $\mathrm{CT}$ in the detection of the PC has not been demonstrated. İn patients with cancer, positron emission tomography (PET/CT) can detect peritoneal carcinomatosis with high specificity. The superiority of PET / CT over CT is clear. PET/CT provides functional information. However, its efficiency in the exclusion of PC is low. It has been reported that the diagnostic accuracy and the negative predictive value increase with the combined usage of MRI and PET/CT. However, both modalities are insufficient in detecting peritoneal lesions below $1 \mathrm{~cm}$ (2). In our clinic for patients 
with PC, CT is routinely performed and PET/CT is performed in case of necessity.

If patients are carefully selected in terms of operability in multidisciplinary oncology councils, advanced age is not an absolute contraindication to perform cytoreductive surgery + HIPEC. In young patients quality of life after CRS + HIPEC is better. Prospective studies are needed to confirm the results in elderly patients $(13,14)$.

HIPEC was used in appendix tumors that spread to adjacent peritoneal surface firstly, subsequently it was used in the treatment of intraperitoneally spread colon and stomach tumors. In the following years, it has been used in gynecological cancers such as intraperitoneally spreading endometrial cancer and ovarian cancer. Currently, it has become the standard treatment for peritoneal pseudomyxoma and peritoneal mesothelioma (15).

Multiple organ resections, longer operation and intraperitoneal chemotherapy duration, neoadjuvant chemotherapy, previous abdominal surgeries and low performance scores put PC patients into a high risk group for complications.In the literature, It has been reported that general morbidity and perioperative mortality after CRS + HIPEC were $12-56 \%$ and $0-12 \%$, respectively. The morbidity rate is related to the extent of the disease, the time until cytoreductive surgery, the number of removed organs, age, perioperative blood loss and operation time. Morbidity and mortality rates in our study are similar to those in the literature. Although acceptable mortality and morbidity rates have been published from mid-volume centers, another efficacious factor on complication rates has been reported as center experience (1). In a study of 100 patients, Moran et al. reported a mortality rate of $18 \%$ in the first 33 cases, 3\% in the second 33 cases, and 3\% in the last 33 cases. They emphasized that the mortality rate decreases with increasing experience and all relevant teams should gain experience (16). The extent of the disease and ensuring complete cytoreduction are the most important prognostic factors on both perioperative surgical and long-term oncological outcomes (17). Despite the low number of patients in our results, the fact that a newly formed team reaches this number within 36 months gives hope for the future. In addition, surgeons leading the team have advanced experience in CRS + HIPEC from their surgical background.
Approximately $10 \%$ of patients with colorectal cancer have metastases in the peritoneal cavity at the time of diagnosis, and $40 \%$ of patients with colorectal cancer develop metastasis during follow-up. Overall survival at 5 years of patients with stage IV of colorectal cancer without treatment is anecdotal, with a mean survival of 6 months. Over the past decade, systemic chemotherapy based on 5-FU + leucovorin \pm irinotecan (FOLFIRI) or oxaliplatin (FOLFOX) has improved overall survival up to 20 months. Advances in biological treatments (such as bevacizumab, cetuximab, panitumumab, aflibercept) have increased the survival expectancy to around 24 months. However, there are few studies specifically evaluating the true efficacy of systemic chemotherapy in patients with colorectal cancer and isolated peritoneal carcinomatosis. Peritonectomy procedures and multivisceral resections, as well as cytoreductive surgery followed by HIPEC, enable better overall survival outcomes. Currently, in the literature, the median survival of patients with colorectal peritoneal carcinomatosis ranges from 12 to 32 months; 1 , 3 , and 5-year survival rates range between $65-90 \%, 18-47 \%$ and $17-30 \%(13,18)$.

The risk of peritoneal metastasis in gastric cancer is approximately $40 \%$, with almost $30 \%$ of patients presenting with peritoneal metastases at the time of diagnosis (19). CRC + HIPEC can improve survival in selected patients with gastric cancer accompanied by peritoneal carcinomatosis. While the madian survival is 3 months with only basic supportive therapy, it is 15 months in patients with complete cytoreduction + HIPEC. Gastric cancer with PC is typically treated with systemic chemotherapy, but its effectiveness is unclear, according to the literature. In three clinical studies, systemic chemotherapy has been shown to increase median survival in metastatic gastric cancer to 7-10 months. In these studies patient populations were heterogeneous due to inconsistent randomization. On the other hand most patients did not have PC Similarly. Preusser et al. reported that the response rates to systemic chemotherapy decreased in gastric cancer with PC. There are no clinical studies that directly compare systemic chemotherapy with the combination of CRS + HIPEC in gastric cancer with PC patients. Two recent studies reported that the median survival of gastric cancer with PC patients treated with the CRS + HIPEC combination were longer than 15 months. Importantly, both of these studies reported that the CC is an independent prognostic factor for survival $(17,20)$. 
Phase 1-3 studies onto prophylactic CRS + HIPEC in T3/ T4 gastric cancer are ongoing in China, America, Germany and France (21). In our study, prophylactic HIPEC was performed to one patient.

The majority of epithelial ovarian cancer (EOC) patients presents with peritoneal metastases and around $75 \%$ will relapse in the peritoneal cavity after successful first line treatment. Therefore, EOC appears to be the ideal candidate for IPDD and remains the best studied indication (22). The timing of HIPEC in ovarian cancer is varies. It could be performed at recurrence (first or subsequent recurrences). Accompanied by CRS, during primary staging surgery, for consolidation following primary surgery and adjuvant systemic chemotherapy, during interval surgery after neoadjuvant chemotherapy, and as salvage therapy. However, its most common use is CRS + HIPEC at recurrent disease. Recently, performing HIPEC during interval surgery after neoadjuvant chemotherapy has also become foreminent (4). CRS + HIPEC was carried out as a second or third step treatment in all gynecological cancers included in our study. The first randomized prospective study of HIPEC in gynecological cancers was published in 2015 by Spiliotis et al. In this study, 120 women with advanced ovarian cancer (FIGO IIIc and IV) who experienced recurrence after primary staging surgery and systemic chemotherapy throughout an eight-year period between 2006 and 2013 were randomized. Patient group divided into two subgroups, only CRS followed by systemic chemotherapy was an arm, CRS + HIPEC and systemic chemotherapy was the other arm. When the results were analyzed, mean survival was significantly higher in the group in which HIPEC was performed (26.7 versus 13.4 months, $\mathrm{p}<0.006$ ). The study also shown that complete cytoreduction is associated with longer survival in parallel with many other studies (23). In the study published by Van Driel et al., 245 patients with advanced stage ovarian cancer were evaluated. HIPEC was performed during interval surgery after neoadjuvant chemotherapy. Following three neoadjuvant chemotherapy cycles, patients were divided into two groups. The first group consisting of 123 patients received CRS and adjuvant chemotherapy, the second group consisting of 122 patients received CRS + HIPEC and adjuvant chemotherapy. According to the results of the study, the mean progression-free survival was 10.7 months in the first group, 14.2 months $(p=0.003)$ in the HIPEC group. Mean overall survival was 33.9 months in the first group, 45.7 months $(p=0.01)$ in the HIPEC group (24).
Peritoneal pseudomyxoma is a clinicopathological syndrome due to the accumulation of abundant mucin $(>90 \%)$ in the peritoneum. It may occur in various primary malignancies that produce mucin $(90 \%$ of appendix tumors, as well as ovarian, colonic, pancreatic and urachus tumors). Histopathological subtypes have a severe prognostic effect. Low-grade peritoneal pseudomyxoma(PP) (common peritoneal adenomucinous) is two times more common than high-grade PP (peritoneal mucinous carcinomatosis). The traditional treatment approach of peritoneal pseudomyxoma is maximal CRS that gives poor results. 5 and 10 years survivals are very low in this approach. The new therapeutic approach is the combination of CRS + HIPEC which gives good results. In a retrospective, multicentric study of 2298 patients which had peritoneal pseudomyxoma, postoperative mortality was 2\%, median survival was 196 months (16 years), and 10 -year survival was $63 \%$. Recently, an international cohort study was published including 1924 patients with PMP, investigating the outcome after CRS with or without HIPEC . It was found that the addition of HIPEC after CRS was associated with a significantly better overall survival as compared to CRS alone with a 5-year overall survival of $58 \%$ versus $46.2 \%$ respectively. The addition of HIPEC did not result in more postoperative complications. Therefore, CRS and HIPEC is proposed as the standard of care in patients with low grade appendiceal neoplasms associated with PMP $(22,25)$. Because of the superiority of these results compared to conventional therapy, the CRS + HIPEC approach has become the gold standard treatment for peritoneal pseudomyxoma. in contrast to peritoneal metastases of colorectal cancers, PCI does not have major effect on prognosis in peritoneal pseudomyxoma $(7,26)$. In our study, one patient underwent CRS + HIPEC due to peritoneal pseudomyxoma. His follow-up continues with disease-free survival of 26 months.

Malignant peritoneal mesothelioma (MPM) is considered as a fatal entity. Three subtypes of MPM have been defined as epithelioid, mixed / biphasic and sarcomatoid. Systemic chemotherapy and surgery provide limited benefit. CRS + HIPEC achieved remarkable improvement in the results of MPM compared to conventional systemic chemotherapy. In a phase II study in which 49 patients underwent CRS + HIPEC, the median survival was encouraging with 92 months.1, 2, 3, and 5-year survival rates were 86\%, $77 \%, 59 \%$, and $59 \%$, respectively. The morbidity rate was $25 \%$, and none of perioperative mortality was observed. In addition, factors associated with improved overall 
survival were previous debulking surgery, absence of deep tissue invasion, minimal residual disease after surgical resection, and being under 60 years of age (27). In another observational study which include 20 patients with peritoneal mesothelioma treated with CRS + HIPEC, the median survival was 29.5 months. 1 and 3 years survival rates were $78.2 \%$ and $46.3 \%$, respectively (28). It has been found that survival is affected by the completeness of cytoreduction and histological subtype $(28,29)$. In our study, two patients were treated due to malignant peritoneal mesothelioma and both patients had PCI scores above 20. One of the patients is followed up in the postoperative 2nd month and the other in the 19th month without recurrence.

Consequently, patients with primary diagnosis of colorectal cancer, ovarian cancer, appendix cancer, gastric cancer (selected cases), malignant peritoneal mesothelioma, peritoneal pseudomyxoma who have peritoneal involvement and/or resectable disease limited to the abdomen should be carefully evaluated in multidisciplinary oncology councils and CRS+HIPEC approach should be considered as the first choice.

\section{Declarations}

The authors received no financial support for the research and/or authorship of this article. There is no conflict of interest.

This study was approved by the Eskişehir Osmangazi University non-invasive clinical research ethics committee with the date of 12.01.2021 and number of 7. The study was conducted according to a recent version of the declaration of Helsinki.

\section{REFERENCES}

1. Topgül K, Çetinkaya MB, Arslan NÇ, Gül MK, Çan M, Gürsel MF, et al. Cytoreductive surgery (SRC) and hyperthermic intraperitoneal chemotherapy (HIPEC) for treatment of peritoneal carcinomatosis: Our initial experience and technical details. Ulus Cerrahi Derg. 2015;31(3):138-47.

2. Aksel B, Karaman N, Yüksel E, Kahraman YS, Doğan L, Gülçelik MA. Sindirim Sistemi Tümörlerinde Peritonitis Karsinomatoza Ne Kadar Öngörülebilir? Acta Oncol Tur.. 2017; 50(1): 7-11

3. Miura JT, Johnston FM, Gamblin TC, Turaga KK. Current trends in the management of malignant peritoneal mesothelioma. Ann Surg Oncol. 2014;21(12):3947-53.

4. Minareci Y, Sözen H, Tosun ÖA, Çelik E, Çeliksoy HY, Baktiroğlu M, et al. Practice of hyperthermic intraperitoneal chemotherapy after cytoreductive surgery in gynecological cancers: Technical detail and short-term results. Türk Jinekolojik Onkoloji Dergisi.21(2):1-7.
5. Sugarbaker PH. Peritonectomy procedures. Ann Surg. 1995;221(1):29-42.

6. Hsieh M-C, Lu C-Y, Chang W-W, Wu S-Y, Hsiao P-K, Liu T-J. Experiences with cytoreduction surgery plus hyperthermic intraperitoneal chemotherapy in Taiwan. Medicine. 2017;96(26).

7. Elias D, Goéré D, Dumont F, Honoré C, Dartigues P, Stoclin A, et al. Role of hyperthermic intraoperative peritoneal chemotherapy in the management of peritoneal metastases. Eur J Cancer. 2014;50(2):332-40

8. Arjona-Sanchez A, Esquivel J, Glehen O, Passot G, Turaga K, Labow $\mathrm{D}$, et al. A minimally invasive approach for peritonectomy procedures and hyperthermic intraperitoneal chemotherapy (HIPEC) in limited peritoneal carcinomatosis: The American Society of Peritoneal Surface Malignancies (ASPSM) multi-institution analysis. Surg Endosc. 2019;33(3):854-860.

9. Oken MM, Creech RH, Tormey DC, Horton J, Davis TE, McFadden ET, et al. Toxicity and response criteria of the Eastern Cooperative Oncology Group. Am J Clin Oncol. 1982;5(6):649-55.

10. Jacquet $\mathrm{P}$, Sugarbaker PH. Clinical research methodologies in diagnosis and staging of patients with peritoneal carcinomatosis. Cancer Treat Res. 1996;82:359-74.

11. Clavien PA, Barkun J, De Oliveira ML, Vauthey JN, Dindo D, Schulick RD, et al. The Clavien-Dindo classification of surgical complications: five-year experience. Ann Surg. 2009;250(2):187-96.

12. Roviello F, Marrelli D, Neri A, Cerretani D, de Manzoni G, Pedrazzani $\mathrm{C}$, et al. treatment of peritoneal carcinomatosis by cytoreductive surgery and intraperitoneal hyperthermic chemoperfusion (IHCP): postoperative outcome and risk factors for morbidity. World J Surg. 2006;30(11):2033-40.

13. Cascales-Campos P, López-López V, Torres-Melero J, Arjona A, Muñoz-Casares F, Barrios P, et al. Survival outcomes in patients aged 75 years and over with peritoneal colorectal carcinomatosis after cytoreductive surgery and hyperthermic intraperitoneal chemotherapy (HIPEC): multicenter study of the Spanish Group of Peritoneal Cancer Surgery (GECOP). Clin Transl Oncol. 2020;22(1):130-136.

14. López-López V, Cascales-Campos P, Schneider M, Gil J, Gil E, Gomez-Hidalgo NR, et al. Cytoreductive surgery and hyperthermic intraperitoneal chemotherapy (HIPEC) in elderly patients. A systematic literature review. Surg Oncol. 2016;25(4):378-384.

15. Morano W, Aggarwal A, Love P, Richard S, Esquivel J, Bowne W. Intraperitoneal immunotherapy: historical perspectives and modern therapy. Cancer Gene Ther. 2016;23(11):373-381.

16. Moran B. Decision-making and technical factors account for the learning curve in complex surgery. J Public Health (Oxf). 2006;28(4):375-8.

17. Glehen O, Gilly FN, Arvieux C, Cotte E, Boutitie F, Mansvelt B, et al. Peritoneal carcinomatosis from gastric cancer: a multi-institutional study of 159 patients treated by cytoreductive surgery combined with perioperative intraperitoneal chemotherapy. Ann Surg Oncol. 2010;17(9):2370-7.

18. Glehen O, Kwiatkowski F, Sugarbaker P, Elias D, Levine E, De Simone $\mathrm{M}$, et al. Cytoreductive surgery combined with perioperative intraperitoneal chemotherapy for the management of peritoneal carcinomatosis from colorectal cancer: a multi-institutional study. J Clin Oncol. 2004;22(16):3284-92.

19. Koemans WJ, Lurvink RJ, Grootscholten C, Verhoeven RH, de Hingh IH, van Sandick JW. Synchronous peritoneal metastases of gastric cancer origin: incidence, treatment and survival of a nationwide Dutch cohort. Gastric cancer. 2021:1-10. 
20. Gill RS, Al-Adra DP, Nagendran J, Campbell S, Shi X, Haase E, et al. Treatment of gastric cancer with peritoneal carcinomatosis by cytoreductive surgery and HIPEC: a systematic review of survival, mortality, and morbidity. J Surg Oncol. 2011;104(6):692-8.

21. Morano WF, Khalili M, Chi DS, Bowne WB, Esquivel J. Clinical studies in CRS and HIPEC: Trials, tribulations, and future directions-A systematic review. J Surg Oncol. 2018;117(2):245-59.

22. Ceelen W, Demuytere J, de Hingh I. Hyperthermic intraperitoneal chemotherapy: a critical review. Cancers. 2021;13(13):3114.

23. Spiliotis J, Halkia E, Lianos E, Kalantzi N, Grivas A, Efstathiou E, et al. Cytoreductive surgery and HIPEC in recurrent epithelial ovarian cancer: a prospective randomized phase III study. Ann Surg Oncol. 2015;22(5):1570-5

24. Van Driel WJ, Koole SN, Sikorska K, Schagen van Leeuwen JH, Schreuder HW, Hermans RH, et al. Hyperthermic intraperitoneal chemotherapy in ovarian cancer. N Engl J Med. 2018;378(3):230-40.

25. Kusamura S, Barretta F, Yonemura Y, Sugarbaker PH, Moran BJ, Levine EA, et al. The Role of Hyperthermic Intraperitoneal Chemotherapy in Pseudomyxoma Peritonei After Cytoreductive Surgery. JAMA Surg. 2021;156(3):e206363.

26. Chua TC, Moran BJ, Sugarbaker PH, Levine EA, Glehen O, Gilly FN, et al. Early-and long-term outcome data of patients with pseudomyxoma peritonei from appendiceal origin treated by a strategy of cytoreductive surgery and hyperthermic intraperitoneal chemotherapy. J Clin Oncol . 2012;30(20):2449-56.

27. Feldman AL, Libutti SK, Pingpank JF, Bartlett DL, Beresnev TH, Mavroukakis SM, et al. analysis of factors associated with outcome in patients with malignant peritoneal mesothelioma undergoing surgical debulking and intraperitoneal chemotherapy. J Clin Oncol . 2003;21(24):4560-7.

28. Chua TC, Yan TD, Morris DL. Outcomes of cytoreductive surgery and hyperthermic intraperitoneal chemotherapy for peritoneal mesothelioma: the Australian experience. J Surg Oncol. 2009;99(2):10913.

29. Aksel B, Sahin H, Sari ME. The Effectiveness of Cytoreductive Surgery and Hyperthermic Intraperitoneal Chemotherapy (HIPEC) in Patients with Malignant Peritoneal Mesothelioma: A Tertiary Center Experience. UHOD. 2018;28(1). 